\title{
MetaPhysische ANFANGSgRÜNDE DER Wissenschaften von Geistigem ZU EINER KANTISCHEN WISSENSCHAFTSLOGISCHEN KRITIK DES Fundamentalnaturalismus und ZU EINER Hegel'schen TheORIE-SKizze
}

\section{Gegnerschaften}

Eine Erfahrung der Beschäftigung mit Hegel scheint es zu sein, dass man nur lange genug warten muss, bis auch die neueste Gegenposition mit ihrem eigenen Programm in einen Zustand der Erschöpfung, der Ernüchterung und der Aporien, gekommen ist: und die /Wahrheit itionen und Theoreme entdeckt. Der Dynamik heutigen Philosophierens entsprechend ist dies ein Prozess in immer kürzeren Wellen. Abstoßung und Neuerschließung folgen einander inzwischen oft in geringen Abständen, zuweilen überlagern sie sich in derselben Zeit.

Das gilt heute in besonderem Maße für den Hegel'schen Gedanken des »Geistes « und den daran hängenden ১Idealismus`. In einem Zustand, der gekennzeichnet ist durch empirische Psychologie, empirische Kulturwissenschaft, >funktionale` Modelle des Gesellschaftsprozesses, Theorien künstlicher Intelligenz und nicht zuletzt Kognitions- und Hirnforschung, kommt es zu einer Renaissance hegelianischen »Geist«-Denkens. ${ }^{1}$ Zum Hochmut dabei besteht freilich kein Anlass. Die Gründe, weshalb man sich vom »Geist « - »Geist« in der Tradition Hegels - abgewandt hatte, sind keineswegs hinfällig geworden.

Es waren mehrere, denkgeschichtlich verschiedene Motivationen, die die Verabschiedung des Hegel'schen Konzepts des »Geistes«, am Ende gar den Anti-Affekt vorangetrieben haben. Allgemein haben die Schübe der Preisgabe sich entzündet an den offenbar massiven Normativitäts-Implikationen sowie, beides miteinander zusammenhängend, dem mit dem Gedanken transportierten Diktat der (philosophischen) Theorie gegenüber aller konkreten und erfahrbaren Wirklichkeitsgestalt: dem Normativen der >Bildung , Abweisung des ungebildeten Geistes, vor allem weil dieser moralisch snicht auf der Höhe` sei; dem Normativen des >Fortschritts`im großen; der Gängelung von Empirie im Historischen, desgleichen in Psychologie; dem Substantialismus im Sozialtheoretischen; schließlich der angesetzten Erkenntnisunmittelbarkeit - dass Geist Geistiges gewahre, ohne dazwischentretende, phänomenaufschließende, vergegenständlichende und konstruierende Akte. Erfasst wurde davon nicht nur das Hegel'sche »Geist «-Denken, bes. in dessen \System «-Gestalt, sondern im selben Zug, ungeachtet der bekundeten programmatischen Offenheit in der Wissenschaftskonzeption, vielmehr wegen ihres De-facto-Vorgehens sowie Topoi ihrer Theoriebegründung, zugleich weithin die im Zeichen des Historischen, Besonderen und Vielfältigen angetretenen >Geisteswissenschaften ${ }^{2}{ }^{2}$

1 Um hier nur eine einzige, prominente Richtung zu nennen, die der Analytischen Philosophie: R. Brandom, Making It Explicit. Reasoning, Representing, and Discursive Commitment, Cambridge (Mass.) 1994; J. McDowell, Mind and World, Cambridge (Mass.) 1994; P. Redding, Analytic Philosophy and the Return of Hegelian Thought, Cambridge (Mass.) 2007; R. Pippin, Hegel's Practical Philosophy. Rational Agency as Ethical Life, Cambridge (Mass.) 2008.

$2 \mathrm{Zu}$ den Restposten philosophisch-idealistischen »Geist«-Denkens im Vorgehen der Geisteswissenschaften vgl. noch immer H.-G. Gadamer, Wahrheit und Methode (1960), Tubingen 1975, 185-240. Wenig überzeugend (und mehr ein inner->geisteswissenschaftliches` Phänomen) sind die Projekte, zu denen dies 
Bei all dem ist auch die Absetzbewegung kein Einheitliches. Es ist eine komplexe herausgebildete Lage, und was diese kennzeichnet, war, und ist, eine Pluralität der Stränge - und sachlich: Dimensionen - des Was-statt-dessen und Wie-anders. Unterschiedliche Dimensionen mit auch jeweils genuinen leitenden Problemen überhaupt.

Einmal gezielt eingeklammert lassen möchte ich hier das aus heutiger Denksicht vornehmlich nur noch wissenschaftshistorisch Bedeutsame: die intern philosophischen Naturalisierungs-Schübe des 19. Jhs. - Naturalisierungen des Geistigen nicht nur qua Religiös-Absoluten, Seelischen (Verhaltens-Charakterlichen), Sozial-Objektiven und Geschichtlich-Kulturellen, sondern zuletzt auch Naturalisierungen des Logisch-Transzendentalen. ${ }^{3}$ Sie drehen im Grunde den kantischen erkenntniskritischen Vorbehalt wieder zurück, errichten für alles Sachgehaltliche, auch alles Phänomen von ,Geistigem〈, eine neue Metaphysik ${ }^{4}$ - sei es Materialismus oder Metaphysik des »Willens«. Eingeklammert sei ebenso, auf der anderen Seite, die Boulevard-Variante der Molekular-Biologie - >die Wissenschaft habe irgendein ,Gen ( $(u$. dgl.) entdeckt, das eine bestimmte geistige (auch emotionale oder moralische) Ausprägung oder Kompetenz unseres Menschseins, d. h. was hierin bisher als Persönlich-Geistiges galt, >steuere،.

Diese erste Problemreduktion, ohne die die propagierte Austreibung des alten Denkens ins Inflationäre geriete, berücksichtigt, lassen sich heute namentlich sechs Stränge bzw. Dimensionen einer Alternativ-Theorie zu »Geist « (d. h. exemplarisch Hegel) - und entsprechendes alternatives Wissenschafts-Programm - ausmachen. (1) Statt interner Prozesse eines Geist-igen, statt der Dimension des Geistigen, als dem, worauf das Denken, Theorie und Wissenschaft, geht, das - anthropologischnatürliche - Ausdrucks- und Bezeichnungs-Wollen des Menschen, und dies geronnen in bedeutungs-tragenden Tatsachen der soziokulturellen Welt: >Symbolk-Tatsachen des Darin-Lebens, Darinsich-Bewegens, Damit-Umgehens. Die Trennlinie des zu Thematisierenden und Begreifenden liegt nicht zwischen Geistigem (als einer zusammenhängenden Wirklichkeit des Schlechterdings-nichtNaturseins), die Trennlinie ist statt dessen zurückverlagert, verläuft zwischen dem Geistigen als den spontan-freien generierenden »Energien des Geistes« (Cassirer) - der Bedeutungs-Gebung - und den geschaffen-dastehenden Symbolisierungs-Wirklichkeiten - im Plural -, als dem eben Teil der 'Es-ist-so<-Welt. ${ }^{5}$ Letzteres dann empirisch beschreibbar, Beschreibung einer jeweiligen Faktizität mittels der in der Theorie explizierten Kategorien des Symbolischen.

(2) Das Geistige, als intelligente Bewusstheit, Klarheit, Diskursivität, rationale Bezüge, aber auch Wünsche und Zwecke, dies ganze Geistige zurückzubinden an ein Vital-Geschehen - ein Dahinter- bzw. Darunterliegendes, von dessen natürlicher Verkörperung auch alle Energien und Impulse entstammten; sei das Geistige als dessen Exponent oder als sich verselbständigender »Widersacher« oder einfach als letztlich unbedeutende (»epiphänomenale«) und allenfalls selbstverliebte Zerebralität, und sei jenes eigentliche Vital-Geschehen individuell oder sozial oder kosmisch. - Man kann dies in einem weiten Sinne die slebens (-philosophische Strategie der Naturalisierung nennen. Ihr gehörte in dieser Perspektive auch, als die negative Variante, ein Denken zu, das wie etwa in der

in der jüngsten Zeit geführt hat: aus einer Selbstverdächtigung heraus, dem hegelianischen Schatten nicht zu entkommen, die radikale Flucht nach vorn anzutreten. Vgl. etwa F. A. Kittler, Austreibung des Geistes aus den Geisteswissenschaften, Paderborn 1980.

3 Letzteres prominent etwa H. v. Helmholtz (vgl. Die Thatsachen in der Wahrnehmung, Berlin 1879) sowie in der faktischen Umwidmung philosophischer Lehrstühle zu empirischer Psychologie und uberhaupt auch theoretisch den verschiedenen psychologistischen Strömungen.

4 Im Übrigen auch mit allem selben Normativen wie das abgelöste idealistische »Geist«-Denken.

5 Wo konsequent, dürfte jener Kosmos der Symbol-Tatsachen - die Macht dieser herrschenden Welt, auch Entzug oder Verschwinden des Subjektiven dahinter - entschieden nicht mehr mit einem geradeheraus angesetzten Begriff des »objektiven Geistes« gefasst werden. 
Nachfolge von Foucault jenes hinter den auftretenden Ausprägungen des Sozial-Geistigen wirkende Eigentlichkeitsgeschehen als die jeweiligen herrschenden >Diskurs «-Mechanismen identifiziert.

(3) Eine dritte dominante Alternative ist, das mit mentalistischen und rationalistischen Konnotationen behaftete Konzept des >Geistigen ( zu ersetzen durch einen prätendiert weniger voraussetzungshaften und idealiter neutralen Leitbegriff: den des >Verhaltens`, Verhalten-eines-Organismus, Verhalten-in-Situation. - Da gibt es sicher unterschiedliche Vehemenz des Bruchs, Bandbreiten der Theorie-Umstellung. Wo nicht mit anderen, ferneren Motivationen überformt, kann dies jedenfalls durchaus Freiheitsgrade dieses Verhaltens ansetzen; und die Prägung auf eine bestimmte Verhaltensweise muss auch keineswegs als behavioristische Konditionierung, einfache Internalisierung eines Rollen-Schemas o. ä. verstanden werden. Was dagegen allemal unter die Neutralität fällt, ist, den Bereich der vordem als >Geist ‘ thematisierten Phänomene nicht mehr nach einem Innen-außen-Modell der Abbildung, Manifestation oder Umsetzung einer korrespondierenden seinseigenständigen Repräsentation zu denken.

(4) Davon abzuheben gilt es einen vierten Strang, effektiv mit zum Teil analogen Umbesetzungen, doch aus anderem Motivationszusammenhang heraus antretend. Es sind die Unternehmungen, die Sachverhalte, die einst als bzw. mittels »Geist« verstanden waren, zu entschränken generell von Aspekten individueller Subjektivität, Intentionalität, auch >Sinnc. Diese einst dergestalt subjekt-zentriert - auf subjektive Ansicht, Absicht und emphatisch >Handlung ‘ ausgerichtet - in Blick gekommenen Sachverhalte seien vielmehr nach Maßgabe funktionaler Groß-Prozesse zu begreifen: eine höhere, koordinierende Quasi->Subjektivität` von strukturellen Wirklichkeiten (etablierte Institutionen usw.), in denen ein jeweiliger >Geist` (funktionaler Geist, Verfahrens-Geist) inkorporiert ist, der über die Horizonte der Individuen hinausgeht und deren - inhaltlich selben - subjektiven >Geist ‘ nicht mehr nötig hat. Ein Denken in Kategorien und Mustern von »Geist « (subjektiv) erscheint hier als alteuropäisches - und heutzutage: nur mehr sentimentales - Kleben am individuellen Handeln und Autorschaft, was angesichts der Eigenprozesse bes. des Gesellschaftlichen zunehmend wirklichkeitsfremd sei.

(5) Das Problem - auch Wissenschaftsproblem - des >Geistes serer alltagspsychologischen Vokabulare. Im alltagsweltlichen Leben hantierten wir zunächst mit bestimmten >mentalistischen (Begrifflichkeiten und Verständnismustern, die sich darböten resp. einstellten (folk psychology). Deren spiritualistischer Sonderkosmos aber, wo nicht ohnehin verfehlte Hypostasierungen, Prozess-Substantialisierungen oder schlicht durch die Logik-der-Sprache erzeugte Chimären, löse sich mit zunehmendem Wissenschaftseinblick - sowie zunehmender analytischer Reflexion, was mit einer Redeweise eigentlich gemeint ist und wie diese verwendet wird Stück für Stück einfach in nichts auf. Das Problem des >Geistes` insofern als etwas, das, generell gesprochen, weithin einer (aus dem Alltag übernommenen) unreflektierten Psychologie geschuldet sei. In einem reifen Weltbild, erst recht einem der Alltagshypotheken entbürdeten Wissenschaftsprogramm, wären viele der klassisch `dem Geist` zugesprochenen Phänomene, Sachverhalte, Ursachenvermögen entweder auf ihren berechtigten Sinn hin zu decodieren oder überhaupt eliminativ herauszufiltern: ihr Sein nur eine küchenpsychologische Vorläufigkeit. ${ }^{6}$

(6) Schließlich die Umstellung auf einen zwar in derselben Linie angesiedelten, doch scheinbar elementareren, weniger idealismus-lastigen und normativitätsbehafteten Terminus überhaupt. Statt einem Konzept aus der philosophischen - und: theologischen! - Tradition der griechischen Sprache (lógos, nóêsis, noûs, u. dgl., vollends pneûma) und dabei vor allem dem hoch konnotationsaufgeladenen deutschen Begriff »Geist«, erst recht statt allen schon mit bestimmten philosophisch-ide-

6 Dieses eliminativistische Programm mit einem Materialismus verbunden bei P. M. Churchland (vgl. Scientific Realism and the Plasticity of Mind, London 1979; ders., The Engine of Reason, The Seat of the Soul, Cambridge (Mass.) 1995). So weit muss man nicht gehen. - Vgl. allgemein S. M. Christensen, D. R. Turner (Hg.), Folk Psychology and the Philosophy of Mind, Hillsdale 1993. 
alistischen Problemfragen verbundenen Begriffen wie »Seele«, »Ich«, »Cogito《, ein neutral, ohne Wertung eines höher und eigentlicher die Ausprägungen übergreifender Sachfeldbegriff: das ist heute allem voran der mit dem neuzeitlichen Unternehmen nüchterner empirischer Erforschung verbundene Begriff »mind « - alles als Ausprägungen bzw. Phänomene von »mind«. »Mind« bedeutet dabei zugleich ein bestimmtes Forschungsprogramm, einen bestimmten neuen Typus davon, der das bisherige Theorievorgehen gewissermaßen umkehrt: analytisch-zergliedernd wie das neuzeitliche Erfolgsprogramm Mechanik, analytisch bis zu modularen Akten, und wo möglich auch experimentell (ggf. gedankenexperimentell) mit Pro- und Kontra-Punkten einer Bestätigung abgestützt; konzeptionell, eine Theorie aufzubauen, von unten. "Mind« bedeutet: von einfachen Beispielen aus, in direkter Prüfung bzw. Rückversicherung mit Gemeinevidenzen, Plausibilitäten und empirischem Wissen, ein Model $\Gamma$ zu entwickeln; und dann zu statuieren, alles andere `Geistiger sei nur ein eben komplexerer Fall desselben - und das werde auf die gleiche Weise demnächst dann auch noch erklärt werden können. ${ }^{8}$

\section{Herausgeforderte Wissenschaft}

(1.) Faktizität der bestehenden Lage ist, noch vor der neuerlichen Renaissance, allemal eine breite Front der Abwendung von einem Denken in »Geist «: mehrerlei Stränge von Kritik und entworfener Alternative. ${ }^{9}$ In diesen Bewegungen-weg-vom-) Geist` spiegeln sich die Phänomen-Dimensionen, Phänomen-Entdeckungen, die das Jahrhundert nach Hegel geprägt haben - Phänomen-Aspekte, die das klassische Konzept des »Geistigen« wohl in der Tat nicht genügend zum Tragen hat bringen können. Es waren die Sach- und Problem-Erfahrungen `Gesellschaft< (Gesellschaft als Mechanismus eigener Regeln und Standards und Dynamik); >Leib،; ২Seele (bes. als seelisch-personales Selbst) und Unbewusstes; Instinkt-Prägungen, archaische Affekt-Muster, auch: mit unserer eben anthropologischen Ausstattung verbundene Funktionen der Situations-Filterung und -Vereinfachung; gewohnheitsmäßige (oder durch Massen-Konstellationen induzierte) Typik des Reagierens-auf-...; andererseits Untersuchungen über soziale und psychologische Deformationen und Pathologien, auch deren eventuelle Rückkorrektur; ferner die Erfahrung >Maschine $\prec$, bes. Fertigungs-Maschine und intelligente Maschine (๖Denk-Maschine $)$; schließlich die Erfahrungsdimension >Information`, oder überhaupt die Bedeutung des Steuer-Codes, der software gegenüber der hardware. Der Mensch, als real lebendes Wesen, zeigte sich als gar nicht der vorrangig >Geist<, der er (philosophisch) geschienen hatte; und das Nicht- und Prä-Menschliche erwies sich zunehmend als gar nicht so un-geistig, gar nicht so weit entfernt, wie klassischerweise philosophisch angesetzt war. Wie immer man die Brisanz und Tragweite der einzelnen Punkte bewertet, der Zustand, das wird auch jede Wiederaufnahme des Hegel'schen Gedankens des »Geistes « zur Kenntnis nehmen müssen, ist eine komplexe wissenschaftliche und auch intellektuelle Gemengelage. Aus jenen Phänomen-Erfahrungen heraus

7 In manchem zunächst vielleicht nur Hypothesen dazu.

8 Mit det entscheidenden indirekten Aussage: Erklärt werden können, wenn für diese Alternative zu »Geist« jetzt schon mal genügend Forschungsgelder fließen. »Mind« ist insofern wesentlich auch ein Forschungsversprechen. Und ein Forschungsförderungspool.

9 Als grundlegende Programme - Programme eines An-die-Stelle-Tretens - sind sie mehr als die klassischen, auch sonst formulierten Vorwürfe gegen den philosophischen Idealismus, exemplarisch Hegel, und seinen Gedanken des "Geistes«. In ihnen fungieren dann freilich, in je unterschiedlicher Zusammenstellung, einige Elemente dessen, was man als jene allgemeinen Topoi kennt - Vorwürfe wie: Rationalitäts-Lastigkeit; ein gemutmaßter Theoretizismus (Einseitigkeit des Theoretischen und Intellektuell-Reflektierten); dem Besonderen, Individuellen und überhaupt dem Einzelphänomen kein Recht zu lassen; die Unterstellung allseitiger `Sinn`-haftigkeit; usw. 
haben sich Begrifflichkeiten, Denkmodelle, epistemische Orientierungen - nämlich an welchen anderen Wissenschaften -, ganze Terminologien und Metaphorik etabliert, und auch die so verschiedenen herausgeformten Alternativprogramme zu »Geist « haben, jedes für sich, ihre unbestreitbare Erschließungskraft, haben einen jeweiligen ganz neuen Erkenntnisertrag entbunden. Auch für den Gedanken des »Geistes « hat denn zu gelten, dass die Distanz gegenüber der Hegel'schen Philosophie und deren Wissenschaftssystem nicht einfach nur borniert oder bösartig ist. Von den Motiven der Skepsis gegenüber dem klassischen »Geist «-Begriff und dem damit verbundenen philosophischen ,Idealismus ist einiges schwer abzuweisen. Und die Wissenschaftslage und der Problem-Druck heute sind nicht mehr die von Hegels Zeiten.

Gleichwohl: das Feld, für das einst das philosophisch fundierte Konzept der Welt des »Geistigen« stand - die einzelnen epistemischen Unternehmungen davon geleitet -, hat sich nicht beruhigt. Es ist nicht nur die Pluralität der an die Stelle getretenen Programme, sondern in diesen fungiert augenfällig auch so etwas wie ein >Geist inkognitor.

Eine Pluralität disparater Auffächerung: denn die herausgeformten Weisen des Statt-dessen sind von Grund auf unterschiedlich und sicher nicht in Eines zu verschmelzen. Divergent sind sie schon wissenschaftsstrukturell und dann auch logisch. Die beiden ersten großen Programme, die symboltheoretische Problemverlagerung - auch Empirisierung - und das >lebens «-philosophische Revirement, bedeuten eine >Verkulturwissenschaftlichung (der Sachverhalte des Geistigen; beim ersten so weit, die Theorieaufgabe - und den Theorieanteil in den Wissenschaftsunternehmungen - tendenziell auf Explikation einer fungiblen Beschreibungsbegrifflichkeit zu reduzieren. Die letzten beiden Programme, die Rückführung des Problems auf (alltagsmäßiges bestrickendes) psychologisches Denken und das Programm, eine Theorie des »mind « von unten aufzubauen, in einzelnen analytischen Modellstücken und empirisch gestützt, zielen faktisch auf eine Austreibung überhaupt des »Geistes«. Und die verhaltensbegriffliche sowie die sozusagen `systemfunktionale « Naturalisierung implizieren eine Einklammerung des Problems; das klassische Wissenschaftssystem, das auf »Geist « (»Geist« philosophisch begründet) hin ausgerichtet ist, leiten sie auf eine Selbstüberschätzung der Rolle des souveränen denkend-handelnden Individuums zurück bzw. eine verfehlte Ich-Fixiertheit bei den Fragen des Sozial-Entscheidenden der Wirklichkeit - die »Geist«-Phänomene aber nicht eigentlich >falsch o oder schimärisch, vielmehr durch eine umfassendere Wissenschaftsperspektive überformt (mit der Begrifflichkeit des >Verhaltens` dabei sogar ein Konzept, das eine ontologische Neutralität wahrt).

Zudem kommen das Theoriedenken und mehr noch die entsprechende Wissenschaftspraxis wohl in all diesen Alternativen nicht wirklich los vom »Geist - los davon, irgendwo im Kern der Konzeption eine Struktur von der Art des ,Geistigen، anzusetzen und dienstbar zu machen: sei es ein >Geist ` des Schöpfertums - d. h. welcherlei Bedeutungen geschaffen und herausgestellt worden sind (zu einer Welt des Symbolischen); ein Gestalt-)Geist` der (wahrhaften, authentischen) Vitalquelle, sonst mit dem slebens (-philosophischen Argument des Dahinter- bzw. Darunterliegenden nur zirkulär das faktisch Zur-Geltung-Gekommene, Sich-geltend-Machende affirmiert; in dem verhaltensbegrifflichen Programm effektiv eine Verschiebung von »Geist« auf héxis, habitus u. Ä. (jedenfalls in Restdimensionen, wo sonst in der Tat alles Verhalten nur als Konditionierung gefasst werden müsste) - und dann eben bald auch so etwas wie shabits of the heart (einräumen zu müssen, d.h. eine ganze sinnere`, nicht nur additive Profilstruktur oder Weise dieses Verhaltens; ein `Geist des Systems, d.h. die in den >Institutionen< der Wirklichkeit inkorporierten Funktionsstandards des Wie und zugleich eine gewisse (Basis-) >Mentalität` der Agierenspole (Individuen, Gruppen, Funktionsträger), ohne die (bzw. deren relative Stetigkeit) der Mechanismus des Ganzen nicht seine Integrationskraft aufrechterhalten könnte; eine - und nur so vom Ganzen abziehbare, rausrechenbare - Logik der Alltagspsychologie; oder beim letzten Programm als bleibendes Kernproblem der Einheitscharakter der modularen Akte sowie der Zusammenhang von robjektiver (Theorie-über-... und menschlicher Binnenerfahrung desselben (>Intentionalität‘, >Qualia‘, >Selbstbewusstsein‘, ...). 
(2.) Wie in dieser komplexen Lage, die Veränderungen des wissenschaftlichen Feldes und schlicht die Gegebenheiten heutigen Denkens ernst genommen, Orientierung? ${ }^{10}$ Ich möchte hier zunächst zwei Schritte der Problemstrukturierung und dadurch -eingrenzung vorschlagen. Der erste ist ein strategischer. Angesichts der strukturellen wie sachinhaltlichen Ungeklärtheiten in den zur Etablierung gekommenen Alternativen muss man die Frage des 》Geistigen « - und dann die eventuelle Verteidigung Hegels - allem voran wohl umgekehrt stellen: Was wäre, was bliebe ein Denken ohne »Geist«? Was geriete aus dem Blick - bzw. ist im gegebenen Fall immer verloren gegangen -, wenn man sich von der Tradition Hegels gänzlich abschnitte? Was verlöre das Denken - und auch die Wissenschaften -, wenn das Konzept des 》Geistes « und damit die in ihm gefassten Einsichten verabschiedet würden?

Das freilich ist gegenwärtig noch in keiner Weise global abzusehen. Zumal auch das allermeiste der Programme des Statt-dessen heute, und erwartbar noch lange, mehr Anspruch denn Wirklichkeit ist. Was aber andererseits eben auch nicht heißen muss, dass die Motivationen hinter jenen Programmen, und der erfahrene Problemdruck, ohne Substanz sind. - Das Vorgehen, das in diesem Gegebenheits-Bewusstsein sich nahelegt, wäre ein noch vor jeder sachmaterialen Entscheidung oder Thesis liegendes gezielt strategisch sich begründendes. Es ließe sich vereinfacht das >Prinzip der stärkeren Wissenschaft ‘ nennen. Nach dieser Maxime gälte zweierlei:

- Was durch andere Wissenschaften - der empirischen Erforschung naturaler Manifestationen nähere Wissenschaften (Psychologie, Sozialwissenschaften, Mentalitätsgeschichte, Gesellschaftsanalyse, Kognitionsforschung, Sprach-/ Symbol-/ Werte-/ Diskurs-/ Interaktionsforschung) - auf verobjektiviert-allgemeine Zusammenhänge gebracht werden kann, muss zunächst soweit möglich dieser empirischen Konkretheit und Breite zugeordnet, d.h. überantwortet werden; Philosophie steht dabei in der begleitenden Rolle - der Rolle der Klärung resp. Begründung der Begriffe und Rolle, wissenschaftstheoretische Reflektiertheit beizusteuern (sowie im gegebenen Fall auch: wissenschaftshistorische Bewusstheit). Oder anders gesagt: Wenn es für denselben Sachverhalt, dasselbe Wirklichkeitsphänomen beides gibt oder sich entwickeln ließe, ist es die Philosophie - sowie die Rede von nicht-naturalem >Geistigem <, , geistigen < Faktoren, deren Bestimmungsmacht - , die in der Beweispflicht ist: was es beiträgt, warum es erforderlich ist, dies nicht >nur< als natural, $d$. $h$. über das natural Erfass- und Erklärbare hinaus auch noch als wesentlich >geistige Dimension, Ausdruck des >Geistigen ‘ zu fassen. - Die eine Seite der Maxime also: Problem- und Phänomenverständnis müssen auf erforschungsweiteste empirische Offenheit angelegt sein. Empirisierung in dieser Weise aber bedeutet zunächst - und unabhängig davon, dass dann eventuell in vorsichtige Terminologien, dass es sich dabei um >Geistiges` handle, transponiert - ein Vorgehen, bei dem nicht zu sehen wäre, worin es sich von dem bei aller anderen Wirklichkeitsnaturalisierung unterscheiden sollte: bedeutet Naturalisierung, naturale Manifestationen. Dies ist ein erkenntnisstrategischer Naturalismus, genauer Basis- oder Primärnaturalismus.

- Andrerseits, zweitens, besteht keinerlei Veranlassung, mit fliegenden Fahnen überzulaufen zum Naturalismus als Wissenschaftsbegründung resp. einem irgend ontologischen Fundamentalnaturalismus, überzulaufen zu einem pathetisch abgeklärt sich gebenden Anti-»Geist«-Denken. Veranlassung mit Sicherheit nicht, solange solches Denken und Wissenschaft nicht eine vollständige gleichgehaltvolle Erkenntniseinsicht beibringt, und das ist, sobald man nicht bei dramatisch inszenierten Kleinbeispielen bleibt, nicht entfernt erwartbar. Bis auf Weiteres ist die Lage jedenfalls, dass je radikaler die Abgrenzung, die unter der Feindformel `Hegel ( gleich auch die ganze alte Philosophie entsorgen will, es desto mehr nur elementarste, zuweilen unglaublich krude und konstruierte Modell-Beispiele sind - und dann lediglich ins Große aufgeblasen.

10 Die Hegel-Deutung im spezifischen Sinne (s. auch Anm. 46) muss in diesem Aufsatz zurückgestellt bleiben. Gehen soll es vorrangig um das allgemeine systematische Problem. 
Bei dieser Abgrenzung - entweder »Geist« oder Natur, d. h. alles Natur - handelt es sich denn um eine Ebenen-Verwechslung. Dass philosophische Bestimmungen, und in der Terminologie von »Geist«, einfach in allem als Letzterklärungen stünden, das gilt ja zum Beispiel schon nicht für das wirkungsmächtige Vorbild Aristoteles, und es gilt auch nicht für die ganze Tradition seit der Aufklärung, die Tradition mit begleitend dem empirischen Wissen über >den menschlichen Geist (psychologia empirica). Wo »Geist«-Philosophie Anderes beansprucht hat, war dies in der Tat hoch begründungsbedürftige Metaphysik (Weltmetaphysik), oder Selbstmissverständnis. Philosophische Bestimmungen vielmehr, und gerade als Bestimmungen von »Geist «, stehen in der Erkenntnis im Wesentlichen auch als Rahmen-Platzhalter: Platzhalter von zu begreifenden Sach-Zusammenhängen, nämlich soweit wie sie nicht in einfache naturale Ursächlichkeiten auflösbar sind, und Platzhalter von betreffendem Problem-Bewusstsein. Philosophie, und eben als Bestimmungen von >Geist<haftigkeit, von Ausprägungen bzw. Prozessen des »Geistigen«, ist das Unterpfand gegen drohende Unterkomplexität sich anbietender `naturalistischer ‘ Phänomeneinschmelzungen. »Geist«, dem vorgeworfen, dass er als Sedativ wirklichen Denkens fungiere, Sedativ des Forschens und vernünftiger Weltaneignung, ist, jedenfalls unter der veränderten heutigen Konstellation, im Gegenteil das, was die Wissenschaft wach hält. Vigilant und selbstreflektiert."

Der zweite Schritt der Problemeingrenzung betrifft Differenzierungen. Einige Seiten, die klassischerweise - und bis heute oft - als Leit- oder Metaproblem fungierten, sollten wohl nicht mit der Kernfragestellung vermengt oder gar identifiziert werden. Mit Sicherheit aber bedeuten sie keinen fruchtbaren Argumentationsgang, wenn sie undifferenziert in ein und demselben Theoriebau mit beantwortet werden sollen, direkt zusammen mit der grundsätzlichen Frage, was »Geistiges « ausmacht. In Titeln und Stichworten: das (alte metaphysische, auch theologische) Leib-Seele-, Körper-Geist-Problem; die generelle Frage nach >Dem Psychischen` (oder desgleichen auch ,Fremdpsychischen $)$ ), wie sie das 19 . Jh. und etwa die Phänomenologie des 20 . Jhs. (bes. deren semantische und deren transzendentale Ausprägung) umgetrieben hat; das Problem des Idealismus überhaupt; ${ }^{12}$ dann die Naturalisierungs-Motivation des 19. Jhs., d.h. diese einfach verlängert - dass so, wie diese Naturalisierung am Gott-»Geist« und dem Gesellschafts-»Geist « begann, dann zum Seelen-»)Geist « weiterging (die innerseelische Wirklichkeit, Seele als psychologische Wirklichkeit), dieselbe Denkhaltung und Erkenntnismethoden dadurch bewährt auf alles auszugreifen sich beauftragt fühlen; oder überhaupt das Thema von Freiheit-und-Determinismus, das von Allgemeinheit-

11 Auch das ist natürlich kein Freibrief für jederlei Rede von »Geistigem《: einen Sonderbereich des 》Geistigen«, was tiefer - und wertvoller - als die oberflächlichen Zusammenhänge der Naturalsicht. Die Platzhalter-Funktion ist nur da berechtigterweise zu beanspruchen, solange philosophische Theorie des »Geistigen« Phänomenfragen der empirischen Erforschung und ein reflektiertes Bewusstsein der wissenschaftlichen Unternehmungen tatsächlich offenhält und nicht vielmehr verstellt. Sollte sich z. B. erweisen, dass ein Denken in »Geist«, bei einer bestimmten Tradition Hegels, starke sachmateriale Präjudizien bedeutet, wäre die Berechtigung - jedenfalls in der betreffenden Hinsicht - sicher nicht gegeben: Präjudizien etwa gegen einen (d.h. sozialöffentlich anerkannten) Eigenwert des Individuellen, gegen eine Sperrigkeit, Integrations-Verweigerung, öffentlichen Ungehorsam aus persönlichen Prinzipien (>moralischen oder kulturell-religiösen Überzeugungen usw.) oder gegen die Sicht des Alltags und der lebensweltlichen Erfahrbarkeit; oder auch Präjudizien einer Zurückstufung, gar Leugnung anthropologischer und biologischer Bedingungsfaktoren, von Unbewusstem (auch kollektiv Unbewusstem), massenpsychologischen Phänomenen usw. - Falls es diese Traditionen gibt - faktisch wohl unbestreitbar -, mit der Konzeption der Hegel'schen Theorie des `Geistigen`hängen sie, so werde ich zu zeigen suchen, alles andere denn notwendig zusammen.

12 Vollends wenn ıIdealismus ‘ als Gesinnungs- oder charakterliche Frage verstanden: als Problem von Idealismus-vs.-) Tatsachen'-Mensch (ggf. -Fatalismus, -Nihilismus oder -Zynismus) oder von >Werte ( oder sSpiritualitätı) vs. materialistische/utilitaristische Einstellungen (des Einzelnen, von Gruppen, des Zeitalters). 
und-Einzelheit - bes. wenn moral- oder sozialphilosophisch, als: Gemeinschaft-vs.-naturales-Ego (bzw. -Ego-Perspektive) - oder das von Erklären-und-Verstehen. Dies Andere alles, von dem die Kernfragestellung entschieden abgehoben bleiben sollte, lässt sich allgemein gruppieren zu zum einen sontologischen ( Fundamentalfragen und, beides freilich weit verschlungen, zum andern wissenschaftslogischen bzw. methodologischen Problemtopoi, vor allem Problemdichotomien. Die Differenzierungen vorgenommen, zeigt sich, womit konkrete Wissenschaft, und ohne sich damit naturalistischen Hau-ruck-Thesen zu verschreiben oder auszuliefern, sich im Grunde nicht belasten muss, zeigt, was ein gehaltvolles Konzept des »Geistigen« über weite Strecken einfach offenlassen kann, allenfalls als angehängte Kontroversfrage mit erörtert. -

Dergestalt Probleme heute entflochten - durch die zwei Schritte der Problemstrukturierung und das Verhältnis von Geistigem und Naturalem entpolarisiert, werden die Konturlinien einer bestimmten Konzeptionshaltung sichtbar. Erste Entscheidung dieses Theoriemäßigen überhaupt wäre, die Kernfragestellung, jedenfalls unter der heutigen in Zuspitzung gekommenen Lage der Denkund Wissenschaftswirklichkeit, weder nach Art der großen ontologisch-metaphysischen (und theologischen) Fragen anzusetzen ${ }^{13}$ noch bei der alltagsweltlichen Zusprechung und Erfahrung eines privaten >Innen<, sie vielmehr gezielt zu lokalisieren im Wissenschaftsproblem: wo und in welcher Weise gerade auch die Wissenschaft - ihre Erforschungsunternehmungen - etwas braucht, das, um nicht in Reduktionismen zu erstarren, als eine Phänomendimension bezeichnet - und dadurch offengehalten - bleiben muss, für die Erklärungen (rein) aus naturalen Determinationen zu kurz griffen. Eine offengehaltene Phänomendimension bei Betreffendem von Mentalem, Bedeutungen-und-Gedanken, Geltungen, Institutionen, skulturellen` Ideen und Orientierungswelten u. A. m. Die Art dieser Theorie-Grundlegung, die daraus folgt, wäre unter Anspielung auf das Theorieprogramm, dessen Reflexion Kant zu verdanken ist, zu fassen als Metaphysische Anfangsgründe der Erkenntnis von Geistigem.

\section{Die Wirklichkeit von Geistigem und seine mögliche Theorie ("Metaphysische Anfangsgründe" materialer Sachwissenschaft)}

(1.) Kant hatte in seinen Metaphysische[n] Anfangsgründe[n] der Naturwissenschaft die Konzeption solcher »metaphysischen Anfangsgründe« entworfen als die Theorie der (jeweiligen) schlechthin elementaren Bestimmungen, von denen ausgehend, auf die sich beziehend, in den uns menschlich grundlegenden Sachfeldern Erkenntnisse sich formen - d. h. in welcher Art (Sach-) Kategorien und welcher (sachlogischen) Weise. ${ }^{14}$ Das Gesamt unserer Erkenntnisse, in je den großen letzten Sachfeldern, ist ein Gefüge aus (allemal empirischem) Wissen von bzw. über Faktisches - synchron als Panorama der Beschreibung sowie diachron als zeitliches Gewordensein, Genealogie, Archäologie (im weitesten Sinne), beides als Akkumulierung der Kenntnisse - und andererseits szientifischer Theorie, die auf allgemeine Zusammenhänge ausgeht. Der Theorie-Pol in diesem Gefüge, das Wissen von Allgemeinem, besitzt dabei eine Bandbreite von empirieerwachsenem Induktions-Wissen bis zu strikter Theorie, die die Wissenschaft erst zur Wissenschaft macht und insofern stets das Ziel - auch real die Tendenz - der epistemischen Unternehmungen sein muss. Wo über Sammlung (und äußere Systematisierung) von Erfahrungs-)Gesetzmäßigkeiten` (Regularitäten, Korrelationen,

13 Wie etwa der Frage der Be-seeltheit oder Frage der Unsterblichkeit (des Unsterblichen in uns), die motivierend und als Entscheidungskriterium hinter dem (klassischen) Modell des Leib-Seele-Problems fungieren.

14 Metaphysische Anfangsgründe der Naturwissenschaft, Riga 1786. - Kant im Folgenden zitiert nach der Ausgabe der Werke von W. Weischedel, Wiesbaden 1956 ff., unter Angabe der Originalpaginierung. 
Ereignistypik usw. $)^{15}$ hinaus, sind stets selbe elementare Sachbestimmungen vorausgesetzt, ohne die das Wissen sich nicht innerlich aufeinander beziehen ließe, und bedarf diese Wissenschaft, um »eigentliche« zu sein, einer Fundierung, aus der sich herleitet, dass die einzelnen Gründe und Konsequenzfolgen und Phänomensachverhaltlichkeiten ein Ganzes der Erklärung bilden bzw. beanspruchen können - Fundierung der begründbaren Notwendigkeit der Aussagen und insofern wirklicher Theorie. ${ }^{16}$ Alle allgemeinere Aussage - geltungsbeanspruchend allgemeiner und sachallgemeiner muss auf gewisse erste, schon immer implizierte oder vorausgesetzte Charakteristika der (sachfeldbetreffenden) Gegenständlichkeit-überhaupt sich stützen (oder darauf hinzielen), die allererst die Darlegung eines Warum stiften bzw. ermöglichen; gewisse erste Bestimmungen auch, damit die Erklärungen zu einem konsistenten Erklärungs-Gebäude zusammenwachsen.

So bedarf jede eigentliche Wissenschaft, d.h. mit Anspruch auf notwendige Allgemeinheit und Theorie, zwischen dem einzeln Inhaltlichen und einer universellen Erkenntnistheorie (Erkenntnislogik) aller begründungsfähigen Wirklichkeitsaussagen einer »metaphysischen « Fundierung »aller übrigen [...] Erklärung «: ${ }^{17}$ jede festgültige Wissenschaft bedarf, vor ihrem Inhaltlichen, nicht nur einer generellen logischen Explikation - einer alles übergreifenden Transzendentalphilosophie -, sondern auch eines (sachfeldbetreffenden) »reinen Teils«. Von »metaphysischem « Status sind die in (jeweiligem) Letzterem darzulegenden Bestimmungen, weil sie nicht durch irgend eine empirische Erkenntnis oder Verallgemeinerung sbewiesen` werden können, vielmehr umgekehrt die Voraussetzungen bilden, damit in der allenthalben anschauungsvielfailtigen Gegebenheits-Wirklichkeit begründbar-gültige Aussagen - und Verallgemeinerungen: die einzelnen Phänomenalitäten als Fallvon-... - sich formen lassen; und damit die besonderen Aussagen in Relationen zu einander - in Gründen, sachlogischer Ordnung und idealerweise Zahlenverhältnissen und Formeln - gebracht werden können: zu einem Wissen über eine grundlegende Phänomenalitätsart der Wirklichkeit. Gerade diese »metaphysischen« Elementarbestimmungen verbürgen, dass weitestmögliche empirische Sacherkenntnis in den Blick kommt und epistemische Unternehmung gerechtfertigt und durchführbar wird. Theorie hat diesen Voraussetzungen aller Erklärung zu gelten - sie zu explizieren -, den Grundlagen, genauer: schlechthin basalen Sach-Setzungen, überhaupt möglicher »Gewissheit« dabei: eine Metaphysik des >Was-es-gibt in dem jeweiligen uns in unserer menschlichen Einrichtung wesentlichen Sachfeld, und wie es erkenntnisdarbietend verfasst ist. ${ }^{18} \mathrm{Zu}$ gehen hat es um eine Metaphysik des Wie einer uns menschlich grundlegenden Phänomenalitätsart und entsprechendem

15 Kants Beispiel für solche aus strukturellen Gründen uneigentliche Wissenschaftlichkeit des Wissens ist die (damalige) Chemie. Vgl. Metaphysische Anfangsgründe der Naturwissenschaft, A V, X.

$16 \mathrm{Vgl}$. Metaphysische Anfangsgründe der Naturwissenschaft, A IV f.

17 »Prinzipien a priori aller übrigen [...] Erklärung ( Metaphysische Anfangsgründe der Naturwissenschaft, A VI). - In Kants Redeweise findet sich, auch in den einzelnen Texten noch einmal leicht verschieden, ein z. T. fließender Übergang zwischen »Metaphysik« und »metaphysischen Anfangsgründen«. Die Bestimmungen als "Metaphysik« aufzustellen ist das Zielideal der Theorie: als ein ganzheitliches System und in strikter Hierarchie der Aussagen; soweit bzw. wo es bei Bestimmungen bleiben muss, die - sachbedingt - in den einzelnen Kennzeichnungen dazu nicht bis zu dieser rationalen Geschlossenheit kommen, handelt es sich, so ließe beides sich sinngemäß voneinander abheben, um betreffende »metaphysische Anfangsgründe«.

18 Dieser »metaphysische« Charakter ist, wie Kant mit einem Blick auf die inner-szientifische Begründung in der Tradition Newtons (Philosophiae naturalis principia mathematica) klarstellt, auch bez. der >Naturı nicht durch formal-operative, das hieße hier mathematische Basisbestimmungen - Konstruktionsakte abzudecken; es bedarf - insofern metaphysisch, nicht (ausschließlich) mathematisch - einer Explikation des Was der Gegenständlichkeit. Vgl. Metaphysische Anfangsgründe der Naturwissenschaft, A VIII f., XXIII f. 
Wie der Konkretisierung zu Erkenntnis-Sachverhalten überhaupt - eine metaphysische Grundlegung unserer betreffenden Einsehbarkeiten.

Die schlechthin grundlegenden, irreduziblen dieser Felder, und nur für dies Letzte bedürfe es falle in die Zuständigkeit - einer Metaphysik, bestimmte Kant als die Ausprägungswirklichkeit dessen, menschlich-mentales Bewusstseins-Wesen zu sein, und als das Feld der materiellen Entitäten und ihres Relationen-Geschehens. Sie definieren - und scheiden - sich über zweierlei grundlegende, nicht weiter reduzierbare Sinnes-Pforten, Weisen möglicher Erscheinungs-Gegenständlichkeit: ${ }^{19}$ die Unmittelbarkeit der Selbst-(Zustands-)Gewahrung (»innerer Sinn«) und, außerdem, auch noch das, worüber die Phänomenalitäten des Außer-uns ihr gediegenes Sein geltend machen (»äußerer Sinn «). ${ }^{20}$ Für das Erstere freilich hatte Kant selber keine parallel gebauten »Metaphysischen Anfangsgründe« ausgeführt, konzeptionell parallel zu denen über die Wissenschaft der äußeren materiellen Natur. Er selber hatte dafür eine anders angelegte Grundlegung des Wissenschaftlichen veranschlagt: neben wenigen, hauptsächlich ein-, d. h. abgrenzenden Bestimmungen im >Paralogismen<-Kapitel der Kritik der reinen Vernunft, ${ }^{21}$ ist es eine auf unsere Selbstbekanntschaft und die soziale Praktik rekurrierende Grundlegung, eine Grundlegung vom herausgebildeten natürlichen Wissen her aufsteigend - Grundlegung zur Metaphysik der Sitten; und dann unter dem expliziten Titel »Metaphysische Anfangsgründe « eine Theorie nur der intrinsischen Normativität des Handlungsvermögens eines, insofern vernünftig, Bewusstseins-Wesens. ${ }^{22}$ Beides setzt einen anderen Fokus der Explikation als bei dem ursprünglichen Programm und folgt auch einer anderen Methode, als sie aus jenem Programm sich ergibt und bez. der äußeren materiellen Natur exemplarisch verwirklicht ist; der Bezug zum »inneren Sinn« hat dort keinerlei analoge Bedeutung mehr zu dem beim »äußeren Sinn« und dessen Phänomenalitäten bzw. Gegenständlichkeiten. ${ }^{23}$

Wenn man sich jedoch nicht Kants eigener Ausführung seiner Überlegungen zur Logik der Wissenschafts-Grundlegung verschrieben sieht, ${ }^{24}$ wären absehbarerweise auch für die Phänomenwirklichkeiten dessen, wofür wir ebenfalls empirische Anschauungsbildungen und Evidenzen haben, was aber in seiner je qualitativen Bestimmtheit nicht einfach unter naturale Kausalitäten - deren Determinationsprodukt - verbucht werden kann, gewisse »metaphysische Anfangsgründe« zu projektieren: eine Theorie des >Was-es-gibt $\prec$ in diesem uns Menschen gleich wesentlichen, irreduziblen Sachfeld des `Geistigen ` und wie die Gestaltungszusammenhänge erkenntnismäßig strukturiert sind,

19 »die zwei Gattungen der Gegenstände unserer Sinne« (Metaphysische Anfangsgründe der Naturwissenschaft, A VIII).

20 Entsprechend der höchsten, der transzendentalphilosophischen Reflexionsebene wäre die Reihenfolge wohl in dieser Weise umzudrehen. In den Metaphysischen Anfangsgründen der Naturwissenschaft steht die auch sonst im neuzeitlichen Denken klassische Dopplung mit Außen-und-Innen und demgemäß äußerer Natur (materielles Geschehen) an erster Stelle. - Anders wiederum in der Kritik der reinen Vernunft, B 274 ff. ('Widerlegung des Idealismus`) bei der Problematik des radikalen ("materialen «) Idealismus, ob es den »äußeren Sinn« in dieser Bewandtnis gar nicht 'gebe«, sondern nur Virtualitäten, zweifelhafte, eben gewohnt-herrschende Einbildungen des Für-real-Nehmens.

21 Kritik der reinen Vernunf, A 341-406, B 399-433.

22 Die Metaphysik der Sitten, mit den beiden Teilen »Metaphysische Anfangsgründe der Rechtslehre« und "Metaphysische Anfangsgründe der Tugendlehre«.

23 Der Grund dieser Gebrochenheit im Programm ist, dass es bei Kant überkreuzt ist mit kognitiv-vs.-Praktisches und die »metaphysische« Grundlegungstheorie bei der Seite der Phänomenalitäten des Mentalen selbst sich de facto von Anbeginn auf das Wollen-Können, genauer: soweit durch Vernunft bestimmt, ausrichtet (oder anders gesagt: beschränkt).

24 Zur Distanz sollte schon seine gerade hier eigentümlich einfließende Descartes'sche Weise der Bestimmung nötigen: eine fast substanzdenken-artige Dualität von »denkendem Wesen « / »ausgedehntem (Materie-)Körper«. Vgl. die formelhafte Klassifizierung Metaphysische Anfangsgründe der Naturwissenschaft, A IV/VIII. 
ja überhaupt wie Erkenntnis sich hier bildet. Die Konzeptions-Idee, dass Wissenschaft einer Theorie der sachfeldbetreffenden »metaphysischen Anfangsgründe « bedarf, ist ein Programm, und dies Programm weist, wenn man den Begriff von »eigentlicher Wissenschaft « nicht blind an das Modell von (neuzeitlicher) Naturwissenschaft und ihres 'Erklärens` oder vollends von mathematischem Mechanismus von Materiehaftem bindet resp. darüber definiert, darauf einschränkt, über Kants eigene Konkretisierung hinaus. Auch für die Phänomenalitäten, den Phänomen-Kosmos des >Geistigen< wären »die [zugrundeliegenden, d. h. implizierten oder vorausgesetzten] reinen Handlungen des Denkens [zu explizieren], welche das Mannigfaltige empirischer Vorstellungen allererst in die gesetzmäßige Verbindung bringt, dadurch es empirisches Erkenntnis, d. i. [allgemeinheitsfähige und ausweisbare] Erfahrung werden kann $\aleph^{25}$ - Explikation der logischen und epistemologischen Charakteristika eines empiriefähigen Begriffs (Kategoriengefüges und Erkenntnisprinzipien) von shat sein Sein nur, wenn ein geistfähiges Wesen ist, das dem dies Sein gibt (resp. dies Sein erfährt)،.

(2.) Was wäre dies? Ich sähe dazu noch einmal zweierlei Dimension einer vorbereitenden Klärung, die dem positiven Aufbau jener Theorie den Weg eröffnen. Die erste Klärung beträfe ein klares Bewusstsein des Verhältnisses zu den einfließenden Sach- und Argumentationsannahmen des Modells, wie es von Kant exemplifiziert ist. Denn es ist nicht nur so, dass Kant eigentümlich schematische, zu rigide Kriterien geltend macht zur Bestimmung ${ }^{26}$ sondern die Schwierigkeiten sind zugleich auch komplexer als das, was er in den Blick hat kommen lassen. Die dominierenden Konzeptionen des ganzen Problemverständnisses, auch die kantische, waren dichotomische (oder mittels Polarisierung sich aufrichtende) ${ }^{27}$ Ihre Frage war, wo - oder im Extrem: ob überhaupt - die Grenze verläuft zwischen dem, was klarerweise und unbedenklich naturaler Erklärung - und naturaler Behandlung - überlassen bzw. überantwortet sein kann oder sogar muss, dem Sog immer weiteren solchen Verständnisses wie Zugriffs, natural gleich Anderem, und dagegen Phänomenwirklichkeiten, bei denen es, weil 'geistig‘, spezifischer Vorkehrungen (und entsprechend Verfahren) bedürfte. Die

25 Metaphysische Anfangsgründe der Naturwissenschaft, A XIII.

26 Neben dem Kriterium, dass der Wirklichkeitsgehalt der Erkenntnis über eine charakteristische Weise unseres Sinnen-Bezugs an uns kommt (bez. der Phänomenalität der äußeren »Natur«: »äußerer Sinn«), sind es im Endeffekt drei weitere: ein korrespondierender Basis-Begriff der >ontologischen` Einheit und Stetigkeit aller Ausprägungen (»Materie«); ein Phänomenalitätstypus alles betreffend Wirklichkeitsgegebenen - für die Theorie bedeutet das eine Grundkategorie, deren Sachlogik sie durchzuanalysieren hat (»Bewegung ); sowie die Mathematisierbarkeit - dass das Viele der Fälle auf generelle ProportionenZusammenhänge und Formeln gebracht werden kann. Auch schon Kants eigene konkrete Theoriebestimmungen bez. des Materiellen und dessen Wissenschaften in den Metaphysischen Anfangsgründen der Naturwissenschaft ist darum wohl keineswegs die argumentationsunmittelbare, letztgültige Durchführung seines Konzeptionsprogramms. Und vollends die abgeleiteten Einschätzungen nach der komplementären Seite des »inneren Sinns « machen die Kriterien nur mehr als schematische Ausgrenzungsgründe geltend: "Noch weiter [...] als selbst Chemie muß empirische Seelenlehre jederzeit von dem Range einer eigentlich so zu nennenden [...W]issenschaft entfernt bleiben.« »Sie kann [...] niemals etwas mehr als eine historische, und, als solche, so viel möglich systematische Naturlehre des inneren Sinnes, d.i. eine Naturbeschreibung der Seele, aber nicht Seelenwissenschaft, ja nicht einmal psychologische Experimentallehre werden « - Letzteres deshalb nicht, weil die elementaren Bedingungen wissenschaftlichen Experimentierens nicht gegeben sind: "weil sich in ihr das Mannigfaltige der inneren Beobachtung nur durch bloße Gedankenteilung von einander absondern, nicht aber abgesondert aufbehalten und beliebig wiederum verknüpfen, noch weniger aber ein anderes denkendes Subjekt sich unseren Versuchen der Absicht angemessen von uns unterwerfen lässt, und selbst die Beobachtung an sich schon den Zustand des beobachteten Gegenstandes alteriert und verstellt.« (Metaphysische Anfangsgründe der Naturwissenschaft, A X f.)

27 Noch vor dem, dass in manchen daraus ein ontologischer Dualismus wurde bzw. sich damit verband. 
Frage geht stets aus - blickt - von der Seite des Naturalen und dessen Kriterien, auch Wissenschaftskriterien.

Diese Weise thematisiert freilich schon aus strukturellen Gründen kaum, vor allem nicht konkret, dass ein ganz entscheidender Teil der Frage ja zunächst einmal darin liegt, wo solche Grenze jedenfalls nicht verläuft. Da nämlich zeigt sich, dass das Problem schwerer und einfacher ist, komplexer und zugleich weniger dramatisch, als im Allgemeinen, d. h. formalerweise, gesehen.

Die Brisanz dessen lässt sich bes. an den heutigen Debatten ersehen. Alles an der Frage der Grenze festzumachen, von Kriterien des Naturalen her dabei angesetzt, hat dort eine symptomatische Finalform hervorgebracht. Um den Punkt des (eventuellen) Übergangs und Nicht-mehr-bloß-naturales-Ereignis-gleich-anderen möglichst scharf zu lokalisieren, streng kategorialerweise, als den Beginn eines Mehr, ist schon das Naturale so weit formalisiert, dass seine Phänomenbildungen und Geschehensvollzüge als >ist Träger von Merkmalen` bzw. >Ereignis eines Informations-Unterschieds ' gefasst sind. ${ }^{28}$ Das (in Frage stehende) Geistige ist da dann aufgespalten in einen Informationsgehalt (oder Akte operativer Intelligenz, an einem Daten-Input), jedenfalls was auch eine maschinelle Intelligenz könnte, und einen genuinen Restposten >menschliches Bewusstheits-Erleben - für den dann die Frage die bleibt, ob nicht auch dies in irgendeiner Weise auf rein natural zu erklärende Bedingtheiten und Prozesse zurückgeführt werden kann. Das hat die Problemsachverhalte von vornherein auf das Schema $>$ Geistiges $=$ Informationsgehalt-plus- $x<$ heruntergekürzt, wobei $x$ für das subjektive Wie-Erleben dieser (Bündel von) Distinktionsinformationen steht - und ob da nicht lediglich aus subjektiver Binnenwahrnehmung ein in Wirklichkeit< normal Naturales sich als scheinbar Besonderes darstellt.

Die Sachverhalte - die Frage - des >Geistigen', das ist aber doch nicht nur, dass (oder ob) in der Welt des generell (>monistisch c) Naturalen auch etwas ist, wozu die 1. Person ein privilegiertes, vorderhand nicht ersetzbares Kennen hat; ${ }^{29}$ ist nicht nur die geistige »Intentionalität « und jenes Problem des »phänomenalen Bewusstseins« bzw. der »Qualia«. Die Ausprägungen der Phänomenwirklichkeit des Geistigen - das sind ja vor allem auch: Kreativität bzw. Innovativität; Äußerungen Anderer verstehen - theoretische und praktische Äußerungen, desgleichen emotionale/affektive Äußerungen und gerade auch Äußerungen des Wie-Erlebens -, sowie sozialer Abgleich (Abgleich unserer Unterschiede); Zusatz- und Um-Besetzungen - nenne man sie `symbolische $<$ - generieren, und umgekehrt verstehen - sekundäre ssymbolische Besetzungen der Bedeutungen, die für unmittelbare naturale Phänomenalitäten stehen bzw. worin diese sich einem Subjekt eingeschrieben haben, d.h. Doppelund Mehrfach-Besetzungen; ${ }^{30}$ ferner Prozesse und Wirklichkeiten der Geltung; schließlich Reflexion bzw. reflexive In-Frage-Stellung - überhaupt: das Offenbare anzweifeln zu können -, Fähigkeit modaler Szenarios und überhaupt Theorie-Bildung.

Andererseits ist die Frage des >Geistigen<, und gerade in dieser Breite seiner Phänomenwirklichkeit, auch nicht einfach ein Sub- oder Konkretisierungs-Problem von objektiv-außenbestimmt vs. subjektiv-selbstbestimmt oder -bestimmungsoffen (und insofern unerschöpflich und inkommensurabel) oder von Determinismus-und-Freiheit (d.h.: bloße Fälle einer universellen determinierenden Kausalität oder dagegen In-seinem-Wesen-und-Identität-unabhängig-davon) u. dgl. Wenn man auf die zwei klassischen Wissenschafts-Kriterien >Erklärung-aus-allgemeinen-Ursachen 〈 und >Voraussagbarkeit< (sowie dann ggf. `Steuerbarkeit`) zurückgeht, dann untersteht auch Geistiges, seine Ausprägungswirklichkeiten, in erheblichen Grenzen solcherart Bedingtheiten (und dementsprechend Wissenschaftszugang). Das sind keineswegs nur die bekannten - eher äußerlichen - Zusammenhänge der Psychophysik; sondern es ist vor allem auch all das uns geistige Wesen als natürliche Wesen

28 Auch schon die Elementarbegriffe >(mentaler) Zustand`, >(mentales) Ereignis`, usf.

29 Es sei denn, man überfrachtete dafür umgekehrt den Begriff des `Erlebens` ins Inflationäre - was die

Frage nur hinausschöbe und im Gegenteil erst recht ins Nebulöse und pauschale Irgendwie brächte.

30 Beispiel: , Kreuzı. 
Ausmachende, angefangen von gewissen >anthropologischen` Konstituentien - Affektprägungen, Massenpsychologie, >anthropologische` Muster des Reagierens - über Sinnes- und Gehirnchemie Beispiel: Hormonales, Verhältnis von Licht (usw.) und unserer `Stimmung`, Psychopharmaka, Drogen - bis zu schlicht Statistischem, dem 'Gesetz der großen Zahl . ${ }^{31}$ Es sind `Regularitäten ‘ von offenkundigen Bedingtheits-Zusammenhängen, wie diese Regularitäten auch in weiten Bereichen der (nicht-geistigen) Natur keineswegs höher - und das heißt: strikter im konkreten Determinierungsweg rekonstruierbar - sind. Erst recht dann gibt es Regelmäßigkeiten, die mit den Einzelnen zu Eigen gewordenen Gründen, > Wert/-Orientierungen, >Stil/-Prinzipien, >Ideen ‘ zu tun haben; oder mit schlicht Rollen, Funktions-Positionen, sozialen Verbindlichkeiten. Geist ist nicht etwa das Indeterminierte. ${ }^{32}$ Sondern der jeweilige Geist, der eine Wirklichkeit bildet, vielmehr ist diese - zu Eigen gewordenen, insofern seine Natur qua Habitus und objektives Sich-Verhalten ausmachenden - Regelmäßigkeiten: das Ensemble oder Profil dieser Regelmäßigkeiten. Es ist ein Feld der charakteristisch geistigen Allgemein-Zusammenhänge - Prozessen, woraus Allgemeinheiten der phänomenalen Wirklichkeit sich ergeben. Jedenfalls, es gibt auf dieser logischen und wissenschaftsstrukturellen Ebene keine klare Trennung zweier Sphären, die sinnvoll die beiden Phänomenkomplexe `Naturwelt / ,Wir Geist $\prec$ bestimmen bzw. abbilden würde. Gerade auch nicht ontologisch, noch in der Weise von Dilthey »Die Natur erklären wir, das Seelenleben verstehen wir «.

Um zum positiven Aufbau einer Theorie zu kommen, wäre daneben noch ein zweiter Horizont der Erweiterung - Bedingungen für eine zureichend komplexe Theorie - in Blick zu nehmen. Er beträfe das Geistige als Geistiges: nämlich wie es auch dafür einige naturale Dimensionen, naturale Übergänge gibt, die mit Sicherheit in eine Konzeption mit eingehen können müssen. So erstens die Entstehung, die Herausbildung von Geistigem - aus Noch-nicht-Geistigem und Prä-Formen; zuletzt Entstehung der Vollgestalt des Geistigen, d.h. der Gestalt auch, die es ist, die einen Prozess der Reflexion über die Phänomene (ihrer selbst wie der säußeren` Natur und deren Kausaldeterminationen) aufmacht und die Theorie entwirft. Dies wäre allgemein die sevolutionäre Dimension nennen. Analog gäbe es zweitens das Faktum der ontogenetischen Herausformung, im Einzelwesen, dessen Wachsen, mit im Blick zu haben: die Entstehungsprozesse in Kindheit und Jugend, und vor allem das Hineinwachsen in eine schon je in bestimmter Weise bestehende Welt von Geistigem, Lernen der (objektiv bekundeten) Weisen-Wie des Sich-Verhaltens, sowie Auseinandersetzung damit; aber auch negativ die Einflussfaktoren von Entwicklungshemmungen, von Deformationen, von Pathologien. Der Titel für diese Dimension könnte lauten: die Erwirkung des Geistigen, und der Kampf des Einzelnen um seinen Geist. Diese Dimension wäre sowohl als anthropologische Verfasstheit, Geschehen der anthropologischen Verwurzelung, zu thematisieren wie auch als Prozess der (darin spezifisch menschlichen) Sozialität. - Und schließlich muss auch sich begreifen lassen, dass, im historischen und kulturellen Blick, Geistiges nicht ein Einheitsphänomen, gar mit innerem normativem Telos, ist, sondern in der Breite von charakteristischen Differenzen seiner Gestalt auftritt. Soweit unser historischer Blick überhaupt zurückgeht und was immer wir kennen an sarchaischen Formen von Lebensweisen irgendwelcher Ethnien, dies alles ist zunächst einmal als Ausprägungen im gleichen Raum des Geistigen zu sehen, als Varianten eines primär Gleichen, nicht in irgendeiner Linie des höher und niedriger, des eigentlicher und dagegen Gestaltung, die noch im >Naturaleren verharrt oder verbleibt. Die Dimension wäre also: skulturelle` Differenzen des Geistigen, und dann die Frage nach den Zusammenhängen dieser Gestalt-Varianz mit möglichen ‘äußeren`, gegebenheits- resp. umfeldcharakteristischen Bedingungs-Faktoren. Was es zu erörtern und begreifen gälte, wäre, wie die (im betreffenden Zusammenleben von Menschen) Phänomenwirklichkeit des >Gei-

31 In gewisser Weise kann man für vieles davon sagen: das, ohne das sonst auch > Werbung ‘ nicht funktionieren - zünden - würde.

32 Oder: das Ineffable, allenfalls subjektiv aus divinatorischer Kraft Spür- und >Verstehbareর. 
stigen im (naturalen) Raum und der Zeit irreduzibel vielgestaltig ist und trotzdem ein Selbes im Geist, Ausformung oder Produkt seiner wesenhaften Konstituierungsprozesse, verkörpert.

Diese mehreren Dimensionen - die evolutionäre, die ontogenetische (bzw. sozialisationstheoretische) und die >kulturelle $\triangleleft$ - bedeuten denn genau keine Erklärungen des Geistigen, potentielle `Erklärungen` im naturwissenschaftlichen Sinn, kausale Rückführung auf (`gesetzliche`) naturale Determinationen. Sie stehen für eine Erweiterung der philosophischen Aufgabe. "Metaphysische Anfangsgründe « müssen dem Rechnung tragen; diese Dimensionen anzuerkennen gibt, recht verstanden, durchaus keine Angriffsfronten, kein Einfallstor der naturalistischen Auflösung des Geistigen. Und ich möchte behaupten: Hier setzt Hegel ein, und dies macht auch das Potenzial seiner Theorie. In Hegel haben wir ein Denken des »Geistes«, das nicht einfach unter der Rubrik `Mentalismus, neuzeitlicher $z u$ verbuchen ist. Hegels philosophisches Denken des »Geistes « ist als eine Theorie »metaphysischer Anfangsgründe « zu lesen, die im Sinne des umrissenen >Prinzips der stärkeren Wissenschaft, d.h. als ein Theorie/Empirie-Verbund mit anderen Wissenschaften (sowie mit Alltagswissen), die reflektiertestmögliche Thematisierung der Phänomene menschlicher Geistigkeit begründet.

\section{Struktur eines Theorie-Raums}

Das Konzept des >Geistigen` scheint ein Gedanke, der, der klassischen Philosophie entstammend, mit schweren Hypotheken belastet ist - und zugleich steht der Begriff für eine Wirklichkeitsevidenz, die sich nicht einfach verabschieden, ersetzen, dekomponieren lässt. Die Erklärungsunternehmungen, die Modellierungen des Naturalen rücken immer weiter heran, stellen Zuordnungstopoi des bisherigen Denkens in Frage: und mit jedem neuen Schritt wird binnen kurzem nur noch einmal bewusst, welche Tiefe und Komplexität der Sachverhalte sdas Geistige` ausmacht und welche Weite, woran sich die Umstellung von philosophischer Thematisierung auf andere Wissenschaften zu entscheiden hätte. Die Programme, unter einem Gesamtkonzept >Aktivität (an Stelle $l$ ) (eine Beobachtungserkenntnis der objektiven neuronalen Vorgänge auf den Weg zu bringen - und das hergebracht $>$ Geistige ` dann als die subjektiv erfahrene oder interpretierte Phänomenalität dem zuzuordnen und daran zu bemessen resp. validieren -, sind nur die neueste, äußerste Gestalt an dieser Erklärungsfront. Der Einspruch, dass Persönlichkeit, Sozialität, >Ideen<-Haben (Von->Ideen〈-bewegt-Werden), ja auch Emotionalität damit nicht entsorgt sind, folgt dem wie ein Schatten.

In diesem Zustand kommt es zu einem charakteristischen Nebeneinander und oft geradezu Schwanken zwischen einem engen und einem weiten Problemblick. Im einen ist es ein enger, der den Begriff des >Geistigen ‘ auf die - jeweiligen - aktuellen (oder erhofften) Möglichkeiten des Natural-Erklärbaren fokussiert; seine Thematisierungen definieren sich durch das, was man die Ignorabimus-Schwelle nennen könnte, ${ }^{33}$ das Problem ist an der Frage der Erklärbarkeit von (menschlichem) Bewusstsein-überhaupt festgemacht. Daneben stehen die Diskurse eines weiten Blicks, der gewissermaßen in der Tradition der Motivationen steht, die etwa prominent Max Weber am Beginn des 20. Jahrhunderts wieder bewusst vom ")Geist des Kapitalismus« (u.A.m.) haben sprechen lassen - das, was in der Philosophie gefasst war, aufgreifend und es in die Unternehmungen der neu entstehenden Sozialwissenschaft einschreibend. ${ }^{34}$ Je weiter hier die Pole auseinander liegen,

33 Nach dem Muster der Wissenschafts-Debatte in der Nachfolge von E. du Bois-Reymond (Über die Grenzen des Naturerkennens, Leipzig 1872).

34 Auch gegen die Wissenschaftslage seiner Zeit, die den `Geist<-Begriff aufs Subjektiv-Psychologische verengt hatte. Vgl. bes. M. Weber, Die protestantische Ethik und der ,Geist des Kapitalismus [1904/05], [leicht überarbeitet] in: ders., Gesammelte Aufsätze zur Religionssoziologie, Bd. 1, Tübingen 1920, 17206 (betreffend zentral 30-62: »Der >Geist des Kapitalismus «). 
desto deutlicher wird das Denk- und Wissenschaftskonzeptionelle der Grenzen von Analogien und Parallelisierungen: dass das Problem des >Geistigen ‘ sicher nicht einfach auf der Linie dessen liegt, was den Erfolg der neuzeitlichen Vernaturwissenschaftlichung ausgemacht hatte. In dieser Linie die Versuche der Zurückführung oder Reduktion des ehedem als >Geist ‘ Gedachten oder Projizierten auf naturale - und entsprechend naturalistisch erklärbare - Sachverhalte, das war das Modell der wissenschaftlichen >Entzauberung`, und daraus speiste sich auch das Pathos. Ihre Programme verstanden sich als der große Weg der Wissenschaft, dessen Konsequenz: mehr und mehr zu Erklärungen zu gelangen für das, was vordem mangels Erklärungsmöglichkeit - oder auch: Erklärungsmut - als geheimnisvolle metaphysische Entität und Wirkungszusammenhänge (〉Geistiges`) erfahren und gedeutet worden war. Es war das Erkenntnisverständnis von Wissenschaft-vs.-Metaphysik.

Die Phänomenwirklichkeit des >Geistigen`, jedenfalls in der relevanten Sachkonkretheit ihrer Ausprägungen, sperrt sich der einfachen Entzauberung, deren unvermittelter Objektivierungsutopie. Dass dem aus prinzipiellen - logischen und epistemologischen - Gründen auch erklärbar so ist, lässt sich von Kant und der in Bezug auf die Seite des Nicht-Materienaturalen erforderlichen Konzeptionsweiterung lernen. Die Neuentdeckung des Gehalts des alten philosophischen »Geist«-Denkens, wie sie gerade in der Lage extremer Erklärungs-Hoffnungen heute wieder auftritt und in den verschiedensten Bekenntnissen zu Hegel sich niederschlägt, ist da einstweilen eher nur Manifestation eines gefühlten Defizits, überspringt den von Kant ins Wissenschaftsdenken eingeführten Schritt. Die Theoriereflexion Kants ernst genommen, ist die Frage, die seit dem Ende des klassischen Idealismus einseitig in eine Richtung zu gehen schien - mit dem Ziel der schließlichen Selbstauflösung - , vielmehr überhaupt wieder offen.

Was die Einlösung einer wirklichen Theorie dazu bedeuten müsste, sei hier abschließend in einigen grundlegenden strukturellen Stücken zu skizzieren versucht: Theorie, die als Explikation der "metaphysischen Anfangsgründe« eines Begriffs des der empirischen Erforschung zugänglichen ১Geistigen zu stehen vermöchte. - Begonnen sei, auch das gehört bei dieser Konzeptionsaufgabe mit dazu, ist Teil des Positiven, mit einer Erkenntnisfrustration.

- Im heutigen Zustand vielfältiger Gegenprogramme hat es seine besondere Bedeutung, den Blick auch auf die Motive zu richten, die einst hin $z u$ einem Philosophieren des »Geistes« gefuihrt haben. Eine reflektierte Position ist nicht ohne ein Bewusstsein davon zu haben, dass diese Motive einen durchaus großen Weg der Klärung durchlaufen haben. Das ist ein Weg nicht nur in den Erörterungen der alten Philosophie, in Differenzierungen an »Geist« als stehend für das Prinzip der Lebendigkeit überhaupt (psychê) ${ }^{35}$ sowie Differenzierungen im christlichen Horizont von "Geist« als dem göttlichen Geist, der ein altes System der menschlichen, der gesellschaftlichen Normierungen (»Gesetz«, »Buchstabe« usw.) aufbricht, der befreit von den Beschränkungen eines erstarrten gesellschaftlichen Zustands und dessen lokalen, alltagsvorgegebenen Horizonten, sondern es sind entscheidende Etappen gerade auch in den subjekttheoretischen Reflexionen des neuzeitlichen Denkens. Nicht nur in Bezug auf alle `Philosophie`, sondern auch in ihrer neuzeitlichen Entwicklung ist die philosophische Theorie des »Geistes « nicht einfach ein Projekt. Die wissenschaftsreflexive Konzeption »metaphysischer Anfangsgründe « - das ist nicht schon eine Explikation der Evidenzen und Akte der >Selbstwahrnehmung ‘, basierend auf einem 2-gliedrigen (empiristischen) Modell von sensations / reflections ${ }^{36}$ oder impressions / ideas ${ }^{37}$, der (empiristischen) Genese unserer einzelmenschlichen Bewusstseinsgehalte, und es ist auch noch nicht das klassische »Geist «-Denken

35 Wie sehr gerade auch Hegel Elemente zumal des aristotelischen psychê-Gedankens aufgenommen hat, vgl. P. Stekeler-Weithofer, »Subjektive Seele und intersubjektiver Geist bei Hegel«, in: Hegel-Jahrbuch 2010, Berlin 2010, 221-239.

36 In der Tradition von J. Locke, An Essay Concerning Human Understanding, London 1690.

37 In der Tradition von D. Hume, A Treatise of Human Nature. Being an Attempt to Introduce the Experimental Method of Reasoning into Moral Subjects, London 1739/40. 
von Descartes, Leibniz oder eben in Kants Ausführungen selbst. Bei der Theoriegestalt, die exemplarisch auf Descartes zurückgeht, formte sich der Begriff durch eine Kombination aus einer 3-gestaffelten Argumentation: Frage nach absoluter intellektueller Evidenz - überhaupt die Fixierung auf die Begründungs-Frage -, durch Kategorisierung aller Seinsphänomene nach dichotomischen Attribut-Alternativen - Region (der Phänomene) des »Denkens« durch kategoriale Aussonderung auch unseres eigenen Körperlichen als Teils des Physikalisch-Mechanischen - sowie dann (Re-) Interpretation der Bestimmungen mittels »Substanz «-Schema ${ }^{38}$ Bei Leibniz ruht die Theorie auf zwei hohen Voraussetzungen einer noch strikter ontologischen Problemklärung: Voraussetzung der Hinordnung von allem auf eine Sinn-Ordnung, die nur in und mittels des Geistigen ist, - und schlechthinnige Transzendierung des Kausal-Mechanischen auf dies Finalursächliche - sowie alles NichtScheinhafte von vornherein als Substanzen und darin als Einheit und Individuiertheit (welche dann in den »Geist«-Substanzen zusätzlich über einen Reflexions-Bezug der Selbst-Habe, Selbst-Präsenz verfügten) angesetzt. Darin ist unter den von Anbeginn aus ontologischen Gründen veranschlagten Theoriekriterien - sowie theologischen Motivation, inwiefern der Geist Abbild des Göttlichen ist bzw. Stellvertreter der Wahrheit in der göttlich eingerichteten Welt - die innere personale Einheit des »Geistes«, und Unabhängigkeit von äußerem Einfluss schlechthin (vielmehr intrinsisch in Akten "ewiger Wahrheiten « sich prozessierend), bereits vorausgesetzt, konzeptionell in die Argumentation eingeschrieben; individuelle persönliche Einheit, Ganzheit und In-sich-Begründetheit (absolute Insich-Bestimmtheit) des »Geistes « steht als bereits vorausgesetztes Beweis-Ziel. ${ }^{39}$

Ein Weg der mehrfachen Klärung mithin, bis zu Kant und schließlich Hegel: dieses denkgeschichtliche Bewusstsein hat heute essentiell mit zu der Frage nach dem >Geistigen` und dem Potential der philosophischen Erbschaften des neuzeitlichen Subjektverständnisses zu gehören. ${ }^{40}$

- Distanz zu den >mentalistischen< Topoi des alten »Geist«-Denkens kann keine Rechtfertigung für irgendeinen ontologischen Reduktionismus sein. Das betrifft den Typus der Theorie - auf welcher Ebene eine Theorie, um stärkstmögliche Wissenschaft zu fundieren, anzusetzen hat. Denn: `Geistiges` ist immer schon da. Sofern unter menschlichen Wesen, gibt es in der (begegnenden) Wirklichkeit immer schon Bedeutungen, Symbolisierung, Formen-des-das-Leben-Lebens, Gelten, Techniken (die es nicht nur mechanisch zu exekutieren, sondern situativ zu >verstehen

38 Vgl. verdichtet in Principia philosophiae, I, 8-11, $52 \mathrm{f}$.

39 Zu Leibniz vgl. Th. Leinkauf, »Überlegungen zum Begriff des >Geistes` bei Leibniz«, in: G. Abel, H.J. Engfer, Chr. Hubig (Hg.), Neuzeitliches Denken. Festschrift Hans Poser, Berlin / New York 2002, 125-143.

40 Dieser erste Punkt hat auch ein charakteristisches Pendant auf der Seite der Kritik, u. z. gerade in den heutigen Linien der Kritik. Dort hat bes. das Schwanken zwischen einem engen und weiten Begriff des `Geistigen` nicht genügend bewusst werden lassen, dass Naturalisierungs-Programme, wo sie mit universellen Ansprüchen auftreten, sich inzwischen schon fast reflexartig daraus begründen, sich selber ein epochales Was-nicht bzw. Was-nicht-mehr zu konstruieren: eine idealtypische Position, was philosophisches 》Geistes«-Denken sei oder gewesen sei. In der Frage des \Geistigen (jedoch, zumal dessen Wissenschaftsfragen, dürfen Konzepte nicht nur daraus leben, sich aus den Schwächen anderer zu begründen, vor allem wenn dies ohnehin eine selbstgezeichnete Schimäre der Alternative, ein hinkonstruiertes Feindbild ist. Das jedoch ist vielerorts eine unselige Tradition. Im angelsächsischen Raum hat sich seit G. Ryle The Concept of Mind (London 1949) - und in einem neuen Schub mit R. Rortys Philosophy and the Mirror of Nature (Princeton 1979) -, in Frankreich mit Merleau-Ponty und Sartre - und bis hin zur `Postmoderne` - verfestigt, in völliger Blickverengung ganz auf Descartes (allenfalls noch Locke) fixiert zu sein: und mit der nochmal und nochmal neuen Widerlegung von dessen Dualismus zugleich auch Kants Reflexionsbeitrag und vollends Hegel erledigt zu wähnen. Überhaupt dürfte das Denkerbe der alten Philosophie des Geistes auf den Prüfstand zu stellen eigentlich wohl nirgends heißen, an relativ schematischen und insofern schwachen betreffenden Ausgestaltungen sein Mütchen zu kühlen. Dies aber ist meist die Theoriestrategie heutiger Naturalisierungs-Programme. 
danken und sIdeenc; und gibt auch andere Wahrnehmungs- und Denk-Subjekte. Hypothesen über die ursprüngliche Entstehung dieser Realität - das genealogische > Warum (-es-das-gibt bzw. -dazugekommen-ist - reichen in ihrer Erklärungskraft bei weitem nicht an das Konkrete der Phänomene heran. Versuche etwa, dies funktional und dergleichen zu erklären, auch entsprechende santhropologische` Begründungen, haben sich noch allemal als schlicht zirkulär erwiesen. Geist vielmehr ist: das Sich-zur-Wirklichkeit-Machen (und -Erhalten) und als solches ein Faktum. Und mit jedem weiteren >geistigen، Akt, >geistigen< Hervorbringung baut er an sich. Er ist das Sich-Fortschreiben; mit jedem seinem Prozess knüpft er an schon Geistiges an, bewegt sich in, reagiert auf einen Kosmos des schon Geistigen - Eigenes wie auch die erfahrene Wirklichkeit anderer Subjekte -, und mit jedem seinem Prozess verändert er seine Substanz.

,Geistiges`, in seiner konkreten Phänomenalität, ist: die Faktizität einer Wirklichkeit. Deren Struktur- und Prozessmerkmalen hat eine Theorie zu gelten und dann erst gegebenen Falles hereinwirkenden Kausalitäten der äußeren naturalen Konstellation. Das >Da ist das im kantischen Sinne "Metaphysische«.

- Analoges gilt zudem in prinzipieller szientifischer Hinsicht und begründet ein gleichsam thematisierungsstrukturelles, methodologisches `Sinnkriterium ২. Eine philosophische Fundierung »metaphysischer Anfangsgründe « muss nicht leugnen, dass Geistiges eine naturale Seite hat, sowohl die physiologische des Neuronalen als auch evolutionär (usw.) oder die ,Verkörperung in Schematades-Reagierens-auf-... Denn wenn etwas eine zweifellose physische Seite hat sowie sonstige naturale Verwobenheit, aber so gut wie alle Bedingtheiten und Zusammenhänge unter den strukturellen Phänomenausprägungen untereinander und von allem unter den betreffenden selbständigen Entitäten sozusagen von-’geistig`-zu-ıgeistig`zustande kommen und nicht wegen der naturalen Manifestation: dann muss, sogar wenn die generelle Erklärungslücke sich einmal schließen lassen sollte, dies, nicht zuletzt auch wissenschaftlich, als eigene Dimension oder Welt des >Geistigen، angesetzt und behandelt werden. Das ist ein Prinzip, Prinzip auch der Wissenschaftlichkeit, das uns anderswo selbstverständlich ist. Sonst müsste man z. B. auch alle inhaltliche Biologie auf (Teilchen-) Physik reduzieren.

- Für die Phänomene dieses `Es-gibt-Geistiges‘ sind - im Sinne zu explizierender »metaphysischer Anfangsgründe « - wohl drei schlechthin prinzipielle Ebenen zu unterscheiden, sachkonkret und geschehensmäßig. Zunächst das `Geistige im Einzellebewesen: wie und in welcher Gestaltung ein einzelnes ansonsten biologisches Wesen zu einem bewusstseins- und geistfähigen Subjekt ${ }^{41}$ wird. Es wäre eine Explikation des komplexen Aufbaus der Prozesse ‘geistiger Kompetenzen und Selbständigkeit in einem natureingebundenen, sinnenbehafteten, bedürftigen Einzelwesen. Diese Theorieebene gälte dem, wie sich die Zustandswirklichkeit `Geistigkeit` durch Auseinandersetzung des subjektivitätsfähigen Wesens - dessen Energien - mit dem Nicht-Geistigen, dessen sprödem Ist, sowie durch schon bestehendes Geistiges - seien es bewusstseinsinterne »Erinnerungen«, Regungen usf. oder andere Geist-Wesen - herausformt. Warum sollte man dies nicht eine Theorie des >subjektiven Geistes` nennen!

- Zum Zweiten das Geistige als eine Welt des 'geistigen Wie der Verhältnisse der einzelnen Geistsubjekte untereinander: die >geistigen` Formen der wechselseitigen Zusprechung von Eigengeistigkeit, des Reagierens-auf-, Umgehens-mit-einander und vor allem, als dessen etablierte (stabilisierende) Metarealität, die >geistigen ‘ Formen der sozialen Reglementierung, ein geschaffenes objektiv Bestehendes der Geltung, die die vielen Einzelnen untereinander als Wirklichkeit setzen und anerkennen - auch als eine feste Wirklichkeit des >Es-ist-so ( $>$ Die-(soziale-)Welt-verhält-sich-soc) voraussetzen bzw. behandeln. Es ist etwas prinzipiell Anderes als bei Tieren, weder biologisch mitgegebene invariante Instinktmuster des Individuums - bei allen betreffenden Artexemplaren - noch (১tiersoziologische $<$ ) Mechanismen, die z.B. die Ordnung in einem Bienen->Volk (hervorbringen.

41 Ich möchte hier bewusst terminologisch unvorsichtig reden. 
Vor allem aber wird dies Geistige nicht genetisch (o. ä.) weitergegeben, sondern ist nur, wenn - und solange - Individuen dies, und zwar in seiner jeweiligen Zustandsbestimmtheit, tatsächlich leben. Als gelebte Geltungen und Anerkennungen wird es zur jeweiligen skulturellen « Umwelt der nachfolgenden, hineinwachsenden Wesen. Warum sollte man die Explikation dieses Was-es-gibt nicht eine Theorie des sobjektiven Geistes` nennen! Theorie auch, die die klare und differenzierte empirische Thematisierung dieser Sachverhalte - und dazu gehört auch: eventuell normative Kritik - aufschließt.

- Zu dem, dass ein Sich-Ausdrücken in Geistigem und Orientierung im Geistigen Wirklichkeit ist und sich in der naturalen Welt, den naturalen Wirkungsverflechtungen erhält oder perpetuiert, gehört - zum Dritten - konstitutiv auch, was und wie die Subjekte jeweils über sich denken. Geist ist immer eine sTheorier. Und dies nicht nur so, wie das heute als Faktum der Entwicklungspsychologie allenthalben mit hereinkommt: als der einmal von jedem (menschlichen) Kleinkind zu lernende Schritt - und als solches auch in allen Kulturen gleich -, ein befremdendes Verhalten eines Anderen $^{42}$ mir in seinem Warum durch die Eigengeistigkeit des Anderen verständlich zu machen, dass der Andere wohl die-und-die Situationsüberzeugungen (sowie Emotivität) hatte abweichend von dem für mich Selbstverständlichen. Das ist der Schritt des Überhaupt, unter Menschen einräumen zu können, dass der Andere aus so etwas wie sseiner (Geistigkeit heraus agiert und empfindet, die mit smeiner (nicht schlechthin identisch ist. ${ }^{43}$ Stets eine >Theorie ist der Geist vielmehr vor allem auch spezifischer: als die etablierten und im sozialen Zusammen selbstverständlich vorausgesetzten charakteristischen (’kulturellen`) Vorstellungen von sich; als die den (jeweiligen) menschlichen Wesen in all ihrem Sinnen und Trachten zueigenen Anschauungen und Gedanken über das, was Geist ist, sein Sein, und worin unterschieden von den Determinationsmächten der Natur und des Anthropologischen, Anschauungen und Gedanken über das, was - ihre - geistige Subjektivität ausmacht und das Verhältnis zu, Stellung in der Naturwelt. Es sind Vorstellungen, mit denen er seine eigenen Erfahrungen schon immer interpretiert - interpretiert als selbsthaft (selbst/nichtselbst-Schwelle) und als Nicht-nur-Natur. Das wird nicht zuletzt gebildet durch die Vorstellungen - das (jeweilige) System der Anschauungen, Gedanken, auch Bilder und Metaphern -, was ein geisthaftes Wesen kann und was es sollte; was ein solches Leben bedeutet - für den Einzelnen oder als Gemeinschaft -, welche Erfahrungen, welches Ringen, welche Krisen; und was ein Verbleiben im (relativ) Naturalen oder Zurückfallen wäre. Eine Explikation der geistigen Vorstellungen vom Geistigen, der Struktur und Subjektbedeutung dieser jeweiligen etablierten >Theorie<: das müsste man doch wohl die "metaphysischen Anfangsgründe« des Was-es-gibt an Phänomenen des sabsoluten Geistes« nennen! Inhaltlich ist, wie immer die geschichtlich->kulturelle، Gestalt dieser herrschenden >Theorie` und an welchem Pol im Feld der Varianzen, dieses Was-es-gibt nicht sinnvoll reduzierbar auf Soziales, Subjektives, erst recht Naturales seiner ansonsten unbestrittenen Bedingungsverflechtungen.

- Das hat, abschließend, zwei nicht unbedeutende Folgeaspekte. Zum einen: Die Entwicklung geht von der Seite des aufgefasst `Geistigen « aus - der Geist, die jeweilige herrschende >Theorie<, gibt etwas als Natur und Andersheit frei; und der Geist auch bestimmt den (empirischen) Geltungsbereich und -modus seiner höchsten Vorstellungen von sich, welchen (menschlichen) Wesen er Vollund welchen er führungsbedürftige Geistigkeit zuspricht, welchen selbständige und welchen unselbständige Subjektivität. Was die erstere Dynamik betrifft, so sind ursprüngliche Welt-Deutungen und -Orientierungen ja stets >spiritualistischer< Art; sie deuten alles Sein als Wirkgeschehen von >geistigen Entitäten, vorgestellten Subjektivitäten-dahinter mit Selbst-Gestaltung, Behauptung-ihrerIdentität und personalem , Wollen<. Erst in einem zunehmenden Prozess haben sich, vom Geistigen

42 Gleichermaßen: ich selber in einem früheren Zustand, wo - aus gegenwärtiger Sicht - ich etwas >nicht wusste, , falsche< Überzeugungen hatte usw.

43 Zur Entwicklungspsychologie dieser »Theory of Mind« s. P. Fonagy, G. Gergely, E. L. Jurist, M. M. Target, Affektregulierung, Mentalisierung und die Entwicklung des Selbst, Stuttgart 2004. 
ausgehend, Phänomenzusammenhänge bzw. Geschehensdimensionen in immer weiteren Wellen als neutrale Kausalitäten der >Natur` ausgegliedert. ${ }^{44}$ Und auch beim Menschlichen ist es eine Dynamik, von den Vorstellungen $\ddot{u b e r}$ Geistiges ausgehend immer Weiteres als eben, wie Hegel den treffenden Ausdruck geprägt hat, zu erfahrende »Prosa des Lebens« dranzugeben und zu behandeln; sowie immer Weiteres als Funktionsprozesse und Reaktionsmechanismen, die wir offenbar mit dem Tierischen teilen. Bis dies gegenwärtig an die Grenze geht, mit diesem Freigeben-als-Natur - dem großen Weg der Einrichtung etablierter (und erfolgreicher!) Praxen naturalen Denkens - sein Eigenes zur Disposition zu stellen.

- Zum anderen: Theorie ihrerseits, alle Theorie, ist Geist. Auch die, die den >Geist $८$ naturalistisch aufzulösen versucht. Auch das ist: eine Theorie. >Geist 〈 ist nicht im Einzelnen von zu erforschenden Bedingtheiten zu >widerlegen', entzaubern o. ä. Die reflexive Distanz durch wissenschaftliche Objektivierung und zu zeigende Kausalitäten betrifft nur spezielle Zuschreibungen, Attribute, Denkmuster, mit denen ein vormaliger magischer Glaube, Religion, Philosophie ${ }^{45}$ oder auch Alltagspsychologie die Dimension des >Geistigen ‘ belegt haben. Im Prinzipiellen aber steht der Gedanke des ,Geistigen،, als philosophisch zu explizierend, als Rahmenplatzhalter für ein nicht einzuschmelzendes Problem-Bewusstsein des Erkenntnisprozesses. Und auch überhaupt: Als Deutung, die ja auch die Deutung ihrer Theorieleistung selbst mit einschließt, unterliegen alle höheren theoretischen Aussagen über das Geistige gewissen logischen Auflagen der Selbstapplikation.

\section{Zeichen der Zeit \\ (Oder: Die Philosophie ist so dumm nicht, wie man ihr unterstellt)}

Es sollte heute klar sein, dass das Gesamt dieser Konzeption, dergestalt umrissen, nicht mit dem identisch sein muss, was als Corpus des Hegel'schen 'Systems - Teil Die Philosophie des Geistes vor uns liegt. ${ }^{46}$ Auch gegen das faktische »Geistes«-Denken der früheren Philosophie setzt der in

44 Das hat auch heute noch, im Endstadium der nüchternen wissenschaftlichen Sicht, einen Restposten: nämlich die »Naturkräfte« ihrerseits. Diese aber werden nicht in einer Theorie »metaphysischer Anfangsgrüne« im Sinne Kants expliziert, sondern diese Problemstelle wird mit einer (mathematisch-symbolischen) Formel, die die Beschreibung dieses Dass - dessen Parameter - abzudecken verspricht, entsorgt.

45 Etwa das Schema der zwei »Substanzen«, körperhaft/denkend, bei Descartes oder die »Monaden«-Identität bei Leibniz.

46 Überhaupt ist auch Hegel in seinem philosophischen Denken des »Geistes« durchaus vielmotivischer, als es die geschlossene Gestalt des >Systems` in seiner Encyclopädie repräsentiert. In gewisser Weise spiegelt sich die systematische Komplexität des Problems in den Etappen und Linien bei Hegel. »Geist « bei Hegel: das ist kein Generalkonzept seines denkerischen >Wollens`, sondern zu konstatieren ist zunächst einmal ein Auf- und Abtauchen des Begriffs. In seinem frühen Denken (sog. , Jugendschriften`) fungiert der Begriff in entscheidender Stellung: in der religionsphilosophischen Thematik als der theologische ruach-/ lógos- /pneuma-Begriff, und im Sinne des Neuen Testaments gegen das "Gesetz«, als das ÄuBerliche und Positive, gerichtet - welches vom "Geist« zu Überwindende Hegel hier zunehmend mit dem kantischen Typus einer Normen-Ethik identifiziert; hingegen ist es bei den gesellschaftlichen und politischen Themen der eher globale »Geist«-Begriff der Montesquieu-Lektüre. In beidem steht »Geist« für: »Leben«, »lebendig« (qua individuell, Organismus u. a.), und in Opposition gegen »erstarrt« oder "mechanisch «. Dann in seinem Schritt in die Philosophie im engeren Sinnen hat Hegel wie eigentlich alle seiner damaligen Orientierungspole signifikant wenig, jedenfalls nicht mit erkennbarem konzeptionellem Gewicht von "Geist « gesprochen; abgesehen von der hart substantialistischen Rede von »allgemeiner Geist« qua holistischer »Sittlichkeit " (wie in der gegen alle Moderne idealisierten Antike). Auf der anderen Seite aber ist es frappierend, wie in Jena binnen weniger Jahre ein Umbau des Hegel'schen >Systems 
diesem Aufsatz basal am Wissenschaftsproblem festgemachte Theorie-Rahmen Bedingungen. Es sind Bedingungen zugleich, damit die heutige Renaissance des Hegel'schen Beitrags in der Theorie des Geistigen nicht unterkomplex und unkritisch bleibt, nicht nur das Spiegelbild und der Gegenstoß gegen ein herrschendes Naturalisierungsversprechen und seinen Positivismus.

Die Konzeption steht für eine Offensive der Philosophie. Eine offensiv auftretende Philosophie, die nichtsdestotrotz selbstkritisch, zumal auch gegenüber ihrer eigenen Tradition in diesem Feld, zu sein vermag. Sie steht gegen die Virulenz von Tendenzen, in den höheren prinzipiellen Themenregionen die wissenschaftliche Erkenntnisrichtung umzudrehen und die Antwort zum Kriterium dafür, was die Frage war oder wäre, zu machen - das Problem des `Geistes` entsprechend den AntwortHandhaben einfach umzudefinieren. Eine Konzeption »metaphysischer Anfangsgründe« dagegen, wie sie sich hier skizzieren ließ, setzt unverhandelbar an dem an, was die Breite der Sachverhalte dieser Phänomenwirklichkeit unserer selbst - und die Tiefenschärfe der wissenschaftlichen Thematisierung sein müsste. Die Frage nach der Frage, offen und differenzierend, ist ihr Erstes.

Was `Geist ist und wie wissenschaftlich zu begreifen: das ist im heute Herrschenden mehr Einstellungsmeinung und Programm denn Wissen und ausweisbare Theorie. Zusehends verschwimmen die Grenzen. Jedes Dilettieren eines Naturwissenschaftlers mit großem Anspruch hier nobilitiert ihn zum »Philosophen«. Sobald jemand mit irgendwelchem positivistisch angekündigten >Die Wissenschaft hat gezeigt, ...< auftritt, ist man bereit, jederlei daraus groß ausgemaltes $>$ Ganz neues Verständnis unseres Geistes! ‘ zu übernehmen, staunend und erschaudernd. Man scheint bereitwillig auf den überall angekündigten Umsturz unseres althumanen Menschenbildes eingestellt. In der Öffentlichkeit heißt es dann immer gleich: $>x y$, Neurowissenschaftlicher und Philosoph, >Genforscher und Philosoph`, ২Kosmologe und Philosoph` und ähnliches.

So schnell sollten wir uns den Begriff - den Reflektiertheitsanspruch - der Philosophie nicht wegnehmen lassen. Mir ist unverstehbar, weshalb man bei den heutigen neuartigen naturwissenschaftlichen Forschungsergebnissen, so vor allem denen zum >Gehirn<, vor Ehrfurcht erstarren sollte. In den meisten Fällen reicht, was die pathetisch gezogenen Folgerungen betriff, schon der Hinweis auf doch bitte ein bisschen Theorie-Bewusstsein, Hinweis auf die wissenschaftstheoretische Naivität solcher >philosophischen< Ableitungen; oder der Hinweis darauf, dass das wenig mit dem zu tun hat, was das tiefere philosophische Problem ist - also Hinweis auf die Naivität einer selbstgestrickten Problem-Definition, die weit hinter dem zurückbleibt, was die Entwicklung des Denkens in zweieinhalb Jahrtausenden als Stand des Problem-Bewusstseins erkannt hat.

stattfand, der alles vom Leitkonzept »Bewußtseyn« (oder anfangs »Sittlichkeit «) auf »Geist« umgestellt hat - wie man dies etwa in seinen Systementwürfen von 1803/04 (GW 6) vs. 1805/06 (GW 8) greifbar findet -, und zwar ohne dass die meisten der Kerntheoreme dabei tiefer verändert oder gar umgestürzt worden wären. - Zwei mit diesen Befunden verbundene Deutungsansichten dürfen, nebenbei gesagt, wohl als falsch gelten (unfruchtbar sind sie ohnehin): dass, nach dem Muster von Dilthey gedeutet, beim »jungen « Hegel mit dem Gedanken des »Geistes« das noch Lebendige und Offene auch seines Denkens selbst verbunden sei, wogegen der Gedanke später in das Korsett des geschlossenen, toten >Systems eingezwängt sei; oder dass Hegel, wie vor allem heute kritisiert, mit genau jener Umstellung auf eine Theorie des 》Geistes« sich all das Problematische, was seit dem 19. Jh. zur Abwendung von seiner Tradition des Denkens gefuihrt hat, eingehandelt habe - doch die berechtigten Kritikpunkte, die Implikationen und Verengungen im Thematischen (s. o. Abschn. I. / II.1.), hängen keineswegs mit dem Konzept des (philosophisch zu explizieren) ,Geistigen ‘ als solchen zusammen, im Gegenteil wäre mit dem (recht verstandenen) philosophischen Gedanken manche Kritik argumentativ noch schärfer zu fassen. - Wieviel von einer Theorie "metaphysischer Anfangsgründe« in Hegel auch so ausgeführt ist und wo er unzweifelhaft auch konzeptionell weiterzuformulieren wäre, muss hier in diesem Text unerörtert bleiben. Desgleichen, ob - an Hegel zu lernen - jenseits der »metaphysischen Anfangsgründe» auch Stücke einer materialen Philosophie des Geistes sich ergeben. Das hätte sich an der Sache zu zeigen. 
Viele der großen neuen Thesen über `Den Geist` sind, wo sie sich der Anknüpfung an die Tradition der Philosophie überhoben denken, wohl eher Zeichen der Zeit. Nassforsches Selbstbewusstsein ist noch keine Theorie. Die Willfährigkeit der Philosophie freilich hat dem meist wenig entgegengesetzt. Bei allen erreichten wie auch entfernt absehbaren neuen naturwissenschaftlichen Erkenntnissen jedoch - die Wissenschaftsunternehmungen in dem, menschlich-mentales Wesen zu sein, werden auch weiterhin, und je grundsätzlicher die Erkenntnisdaten, desto mehr, der Philosophie als Sachwalterin eines komplexen Problembewusstseins bedürfen. Bedürfen auch, um nicht in einem Positivismus der Fakten unterzugehen. Hegel, im Sinne einer Konzeption »metaphysischer Anfangsgründe« der Wissenschaften vom Geistigen gelesen, einer Konzeption, die die Ebenen des Wissenschaftlichen zu unterscheiden lehrt, ist da kein schlechter Pol, um eine solche erforderliche Theorie, die das Empirische vorbehaltlos zur Kenntnis nimmt und ihm breit Rechnung trägt, doch um gleichwohl nicht in den Sog eines sich aufplusternden Naturalismus zu geraten, herauszuarbeiten.

Das Thema ,Geist`, die Weise des wissenschaftlichen Diskurses darüber, ist heute Index des Stands oder Stadiums unserer Wissensgesellschaft.

\author{
Rainer Adolphi \\ Technische Universität Berlin \\ Institut für Philosophie ( $H$ 72) \\ Straße des 17. Juni 135 \\ D-10623 Berlin \\ rainer.adolphi@tu-berlin.de
}

